\title{
Les formations à Piliostigma en zone sahélo-soudanienne du Burkina Faso: Etat des peuplements, dynamique de la régénération sexuée et pression anthropique
}

\author{
Barthélémy YELEMOU*, Georges YAMEOGO, Babou André BATIONO, \\ Rasolodimby Jeanne MILLOGO et Victor HIEN \\ Institut de l'Environnement et de Recherches Agricoles (INERA) INERA-Saria, Programme Gestion des \\ Ressources Naturelles et Systèmes de Production, BP10 Koudougou. \\ *Auteur correspondant; E-mail: yelbarth@yahoo.fr
}

\section{RESUME}

Les formations à Piliostigma au Burkina Faso connaissent une forte adoption dans les systèmes agrosylvopastoraux. Or, il n'existe pas d'étude sur la dynamique et la structure des peuplements de ces ligneux. La présente étude permet une analyse comparée de la réactivité des Piliostigma face aux conditions environnementales. Suivant un transect allant du Nord au Sud du pays, des formations à Piliostigma (de 20 à 30 ans), ont été déterminées. Dans chaque peuplement, des placettes de 30 m x 30 m ont été installées. Dans la placette de $900 \mathrm{~m}^{2}$, tous les individus de Piliostigma ont été inventoriés. Les paramètres mesurés sont la hauteur du pied, la grosseur du tronc, le diamètre du houppier et la pression anthropique. Dans les placettes, deux sous relevés de $5 \mathrm{~m}$ x $5 \mathrm{~m}$ ont été effectués pour l'appréciation de la régénération. Les jeunes pieds sont dénombrés par espèce et rangés selon 03 classes de hauteur. La classe de hauteur] 100- $200 \mathrm{~cm}$ ] contient la plus forte proportion des Piliostigma quelle que soit la zone phytogéographique, tandis que la classe ] 200- $300 \mathrm{~cm}$ ] marque le début du déclin démographique. La population de Piliostigma diminue grandement à partir de la classe de houppier ] 200-300 cm] à d $>400 \mathrm{~cm}$. Piliostigma présente une faible régénération sexuée qui varie selon les zones phytogéographiques. La classe [0-20 cm[ est généralement la mieux représentée dans les différentes zones. La pression anthropique est diversifiée et forte sur les peuplements de Piliostigma.

(C) 2012 International Formulae Group. All rights reserved.

Mots clés: peuplements, Piliostigma, croissance, pression anthropique, régénération.

\section{INTRODUCTION}

Les zones soudano-sahéliennes sont une mosaïque de faciès aux potentialités sylvicoles différentes (Bationo et al., 2001). C'est dans ces milieux que doivent être récoltées les informations sur les exigences des espèces forestières pour mieux définir les opérations sylvicoles à appliquer (Bationo et al., 2005). Au Burkina Faso, la physionomie de la végétation est marquée par une présence continue du Nord au Sud, des espèces du genre Piliostigma (Yélémou, 2010).

Les espèces de Piliostigma, Piliostigma reticulatum (DC) Hochst. et Piliostigma thonningii (Schumach.), apparaissent de plus en plus dans les champs où elles sont épargnées suite à une dégradation sérieuse, en terme de diversité et de densité des ligneux (Thiombiano et al., 2003). Ces espèces de nos jours sont victimes de leurs nombreuses potentialités. Elles font 
l'objet d'exploitation importante et variée aux champs pour le paillage des zones encroûtées (Yélémou et al., 2007) et leur rôle dans le stock du carbone du sol (Lufala et al., 2009 ; Dossa et al., 2009; Diedhiou et al., 2009 ; Yélémou 2010). En pharmacopée, toutes les parties de ces plantes sont utilisées (Yélémou et al., 2007 ; Kaboré et al., 2007). En outre, ces plantes font l'objet de beaucoup de pression en milieu paysan pour la qualité des fibres (Yélémou et al., 2007) et la valeur fourragère des gousses pour le bétail (Zoundi et al., 2004). Confrontées à la pénurie et à la cherté des aliments pour bétail, les populations rurales se tournent de plus en plus vers les gousses de Piliostigma. Ces gousses écrasées sont mélangées au son de maïs ou du sorgho et servent d'aliment de bétail. Sous la forme farine, ces gousses font de plus en plus l'objet de commerce sur certains marchés locaux du plateau central du Burkina Faso. Espèces à fort potentiel de multiplication végétative, les espèces du genre Piliostigma, s'éloignent de plus en plus des concessions. En effet, les populations locales constatent qu'il faut de plus parcourir de longues distances pour obtenir de longues fibres de ces espèces (donc sur de grands individus). $\mathrm{Au}$ regard de l'importance des Piliostigma pour les différents groupes ethniques au Burkina Faso, il apparaît nécessaire pour leur gestion, de mieux connaître l'état des peuplements, leur dynamique, préalable à la sylviculture des espèces forestières locales en zones soudanosahéliennes.

\section{MATERIEL ET METHODES \\ Site d'étude}

La zone d'étude s'étend entre les latitudes $10^{\circ} 80$ Nord et $14^{\circ} 30$ Nord et les longitudes $1^{\circ} 30$ Ouest et $2^{\circ} 97$ Ouest (Figure1). Elle concerne, au Nord les provinces du Yatenga et du Passoré, au Centre la province du Boulkiemdé et au Sud les provinces du Ziro et de la Sissili. Cet espace est constitué du Nord au sud par un secteur sub sahélien, un secteur Nord soudanien et un secteur Sud soudanien (Fontes et Guinko, 1995). La région Nord de notre zone d'étude, qui comprend la province du Yatenga et celle du Passoré, est soumise à un climat soudanosahélien. Les précipitations y sont insuffisantes et irrégulières avec une pluviométrie annuelle de l'ordre de 600 à 700 $\mathrm{mm}$. La province du Boulkiemdé, partie centre de notre zone d'étude, est soumise à un climat nord soudanien avec une pluviométrie annuelle moyenne comprise entre 600 et 900 $\mathrm{mm}$. La partie Sud comprenant le Ziro et la Sissili, connaît un climat de type sud soudanien, avec une pluviométrie moyenne annuelle supérieure à $900 \mathrm{~mm}$. La saison pluvieuse dure 6 à 7 mois. Deux principaux types de sols se rencontrent dans notre zone d'étude : Dans la partie nord, on distingue les lithosols sur cuirasse occupant 33,3\% de la région et les sols ferrugineux tropicaux lessivés ou appauvris couvrant 39,9\%. La partie Centre et Sud sont constituées à 91,9\% de sols ferrugineux tropicaux lessivés ou appauvris. La zone d'étude est caractérisée par quatre types de végétations: De la province du Yatenga à celle du Passoré, on distingue une brousse tigrée ou fourré tigré caractéristique du climat sahélien, une savane arbustive s'étendant sur la grande partie de la zone et une savane arborée. Du Centre au Sud, on a une savane arbustive qui est la plus dominante du point de vue superficie. Elle se caractérise par des formations mixtes d'arbustes ne dépassant pas 7 mètres de hauteur. Enfin, dans la province du Ziro, on a une savane arborée.

\section{Méthode d'étude \\ Echantillonnage et inventaire}

L'état des peuplements à Piliostigma a été apprécié à travers un inventaire des formations à Piliostigma, suivant un transect allant du Nord au Sud du pays et traversant les différentes zones phytogéographiques où on rencontre le genre Piliostigma. Ces différentes zones sont: la zone sub sahélienne, la zone nord soudanienne et la zone sud soudanienne. Le plan de sondage adopté est un échantillonnage stratifié. L'existence de peuplement à Piliostigma est un préalable à l'installation des relevés. La répartition des 


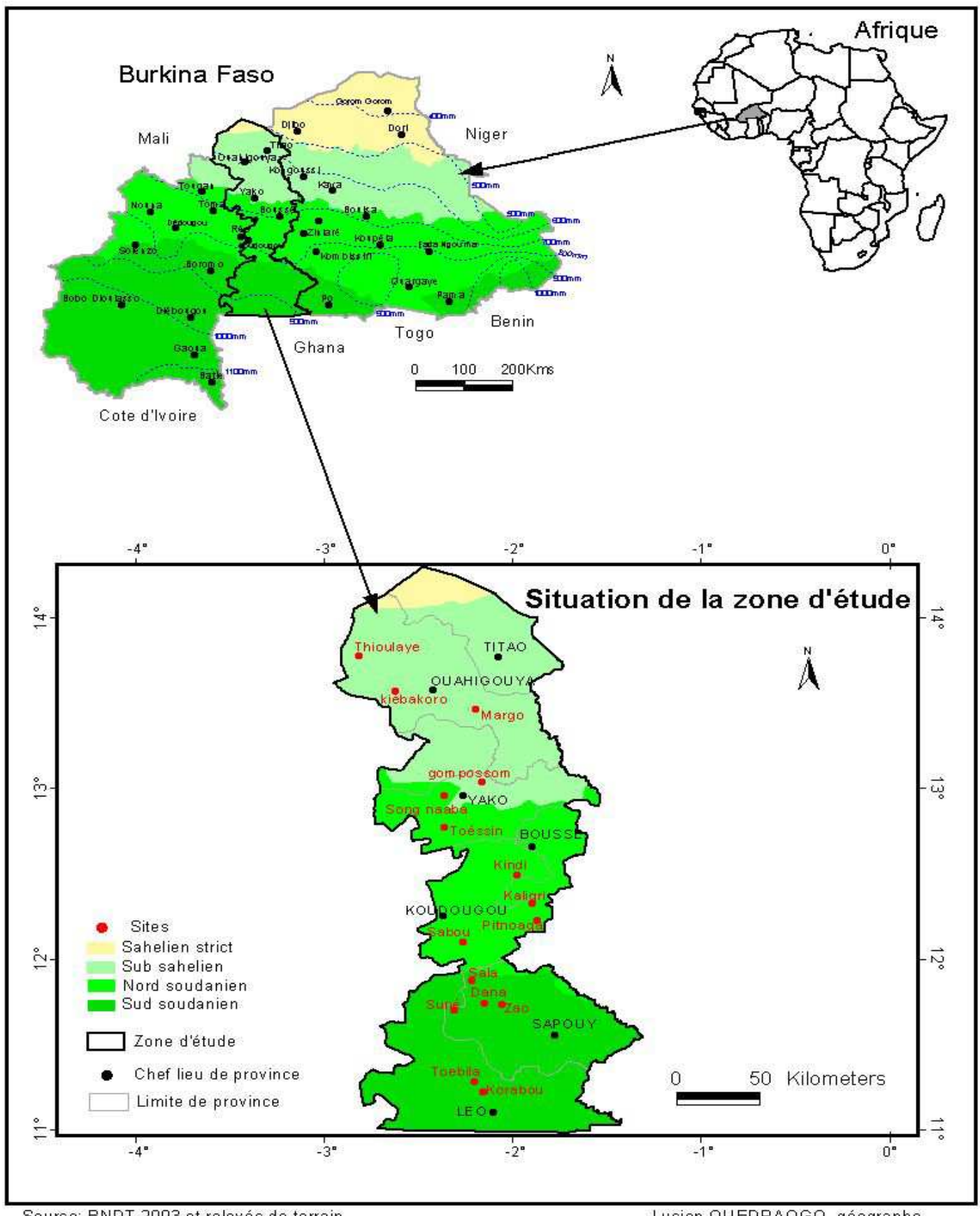

Source: BNDT 2003 et relevés de terrain

Lucien OUEDRAOGO, géographe

Figure 1: Situation géographique de la zone d'étude. 
sites de relevés dans les différentes zones phytogéographiques est faite de façon aléatoire. L'inventaire de la végétation a été conduit vers la fin de la saison des pluies (septembre), période où il $\mathrm{y}$ a moins d'agression sur les jeunes plantules et où les parties aériennes des plantules sont visibles. L'âge des peuplements de Piliostigma varie de 20 à 30 ans et leur densité est donnée par le Tableau 1. L'unité d'échantillonnage est un carré de $30 \mathrm{~m}$ x $30 \mathrm{~m}$ soit, $900 \mathrm{~m}^{2}$, superficie qui tient compte des réalités pratiques des formations de savane (Thiombiano, 1996; Ouédraogo, 2006). L'évolution des peuplements adultes de $P$. reticulatum et de $P$. thonningii dans leurs différentes zones écologiques a été appréciée par des mesures dendrométriques et la collecte d'informations sur le site et la pression anthropique. Sur chaque individu, les mesures ont porté sur le nombre de tiges, le diamètre à la base (à $5 \mathrm{~cm}$ $\mathrm{du}$ sol), la hauteur totale et le diamètre du grand houppier et du petit houppier. L'état d'anthropisation des Piliostigma a été aussi apprécié. Le facteur anthropisation a été évalué à travers de simples observations, à deux niveaux (arbre et site) par les paramètres suivants : trace de coupe à la base, écorçage, taille (recépage), pâturage des animaux, feu de brousse. L'échelle d'intensité de ces agressions a été fixée de 1 à 3 ( 1 = faible ; 2 = moyen ; 3 =élevé). Dans la parcelle de relevé de $900 \mathrm{~m}^{2}$, deux sous relevés de $5 \mathrm{~m}$ x $5 \mathrm{~m}$ sont effectués pour la strate juvénile des Piliostigma. Nous avons considéré comme régénération les individus dont le diamètre à la base (au plus à $5 \mathrm{~cm}$ du sol), est inférieur à $1 \mathrm{~cm}$. Car nous avons constaté au cours de nos travaux sur la phénologie, que certaines tiges de $P$. reticulatum de diamètre compris entre 1 et $2 \mathrm{~cm}$ portent des fleurs, donc atteignent le stade de maturité. Dans chaque placeau, les jeunes sujets de Piliostigma sont dénombrés par espèce et rangés selon les classes de hauteur d'intervalles suivantes : [0- 20[; [2040[ et supérieur à $40 \mathrm{~cm}$. Une excavation du sol sous certains pieds a été effectuée pour mieux cerner l'évolution des plantules de
Piliostigma. Au total, 80 relevés de $900 \mathrm{~m}^{2}$ et 180 relevés de $25 \mathrm{~m}^{2}$ ont été effectués.

\section{Analyse des données}

L'état des peuplements des Piliostigma a été évalué par l'interprétation des histogrammes de distribution des effectifs d'individus en 06 classes de hauteur ([0-100 $\mathrm{cm}$ ], ] 100-200 cm], ]200-300 cm], ]300-400 $\mathrm{cm}$ ] 1400-500 cm] et $>500 \mathrm{~cm})$ et 05 classes de largeur du houppier ([0 -100 cm ], ]100-200 $\mathrm{cm}$ ], ]200-300 cm], ]300-400 cm] et > 400 $\mathrm{cm})$ de la représentativité des espèces par zone écologique, de même qu'à partir du degré d'anthropisation. La dynamique de la régénération des formations à Piliostigma a été évaluée par une analyse en trois (03) classes de distribution [0-20 cm [; [20-40 cm [ et sup. à $40 \mathrm{~cm}$. Des moyennes de régénérations ont été calculées par site et par zone phytogéographique, des tests LSD à une variance ont été effectués pour comparer les différentes valeurs de régénération $(\mathrm{P}<0,05)$. Les liens de dépendance entre les différentes classes de régénération (corrélation de Pearson) ont été aussi établis. Les logiciels Excell 2000 et Statistica version 6.0 ont été utilisés.

\section{RESULTATS \\ Etat des formations à Piliostigma}

La hauteur moyenne des pieds de $P$. reticulatum croît sensiblement de la zone Sud soudanienne à la zone Sud sahélienne (Tableau 2). Les plus grands individus de $P$. thonningii sont situés dans la zone Sud soudanienne, aire de prédilection de l'espèce. Dans une même zone phytogéographique, $P$. thonningii a une hauteur moyenne plus élevée que celle de $P$. reticulatum. Les formations de $P$. reticulatum dans la zone sub sahélienne présentent les plus importants diamètres à la base. Les zones nord soudaniennes et sud soudaniennes ne présentent pas de différence statistique de diamètres moyens à la base $(\mathrm{P}>$ 0,05). $P$. thonningii ne présente pas de différence sensible de diamètre à la base d'une zone à une autre. 


\section{Evolution des populations de Piliostigma selon les classes de hauteur}

La distribution des populations de $P$. reticulatum suivant les classes de hauteur, montre une augmentation progressive des proportions d'individus jusqu'à la classe ] 100 -200], chez les deux espèces de Piliostigma, dans toutes les zones phytogéographiques (Figure 2). Après une faible installation des espèces du genre Piliostigma, marquée par une faible proportion des individus de taille $\leq$ $100 \mathrm{~cm}$, on assiste à une augmentation démographique importante (50\%) à la classe] $100-200]$.

A partir de la classe de hauteur ] 200300], il y a une diminution progressive des proportions des individus des deux espèces. L'évolution en hauteur du genre Piliostigma est faible au-delà de ]100- 200]. $P$. reticulatum évolue mieux en zone sub sahélienne tandis que $P$. thonningii, possède plus de grands sujets en zone sud soudanienne. La classe de hauteur supérieure à $500 \mathrm{~cm}$ est caractérisée par une très faible représentativité démographique, quelles que soient l'espèce et la zone phytogéographique. Chez le genre Piliostigma, il existe une corrélation élevée (89 à 99\%), entre les différentes classes de hauteur des deux espèces confondues. La corrélation la plus faible (89\%) est obtenue entre les classes de hauteur ] $100-200]$ et ] $200-300]$. Ces classes de hauteur caractérisent l'évolution structurale des peuplements du genre Piliostigma. En effet, la classe ] 100-200] contient la plus forte proportion de $P$. reticulatum et $P$. thonningii quelle que soit la zone phytogéographique, tandis que la classe suivante (] 200-300]), marque le début du déclin démographique des peuplements des deux espèces.

\section{Piliostigma et évolution du houppier}

La distribution des populations de Piliostigma suivant les classes de houppier met en évidence une diminution importante des individus des deux espèces au niveau des grandes classes de houppier, ] 200 -300] à la classe $\mathrm{d}>400 \mathrm{~cm}$ (Figure 3). En zone sud soudanienne on a une bonne représentativité démographique $(47,4 \%)$ de $P$. reticulatum, dans la jeune classe de houppier $(\leq 100 \mathrm{~cm})$, alors que la zone sub sahélienne, est marquée par une faible proportion de jeunes houppiers $(13,5 \%)$. Il y a donc en zone sub sahélienne une faiblesse du renouvellement de $P$. reticulatum, qui devra être mis en relation avec le mode de gestion de l'espèce et les facteurs climatiques. Pour les classes de houppier $\leq 100 \mathrm{~cm}$ et ] 100-200], en zone sud soudanienne, la population de $P$. reticulatum est stationnaire alors que celle de $P$. thonningii double de la classe $\leq 100 \mathrm{~cm}$ à la classe ] 100-200]. En zone nord soudanienne, les proportions de $P$. reticulatum et $P$. thonningii, doublent de la classe $\leq 100 \mathrm{~cm}$ à celle de ] 100-200]. D'une classe de houppier à une autre, l'évolution des populations des deux espèces de Piliostigma, montre une grande dépendance. La corrélation entre les différentes classes de houppier varie de $91 \%$ à $99 \%$.

\section{Structure et potentiels de régénération des formations de Piliostigma}

$P$. reticulatum et $P$. thonningii, présentent une faible densité de régénération quelque soit la zone phytogéographique considérée (Tableau 3). La zone nord soudanienne présente une meilleure régénération pour l'espèce $P$. reticulatum, tandis que pour l'espèce $P$. thonningii, la meilleure régénération est obtenue dans la zone sud soudanienne. L'évolution de la densité des plantules selon les différentes zones phytogéographiques met en évidence l'importance des latitudes sur la régénération des espèces du genre Piliostigma. La distribution démographique de la régénération en classes de hauteur selon les zones phytogéographiques, chez les Piliostigma, donne des histogrammes d'allures diverses (Figure 4). En zone sub sahélienne, l'analyse de variance, à une variable avec le modèle $\mathrm{LSD}$, révèle une différence significative de densité $(\mathrm{P}<0,05)$ entre les plantules de 0-20 $\mathrm{cm}(12,57$ pieds /ha) et les plantules de 20-40 $\mathrm{cm}(1,71$ pieds $/ \mathrm{ha})$, de même qu'avec celles 
supérieures à $40 \mathrm{~cm}$ de hauteur (2,29 pieds /ha). Entre les plantules de $20-40 \mathrm{~cm}$ et supérieurs à $40 \mathrm{~cm}$, il n'y a pas de différence significative $(\mathrm{P}>0,05)$. Il y a une importante prédominance des plantules de $0-20 \mathrm{~cm}$. En zone sud soudanienne, la même tendance est observée pour $P$. reticulatum. Pour $P$. thonningii, la différence est très significative $(\mathrm{P}<0,001)$ entre les différentes classes de hauteur soit 43,09 pieds /ha pour $0-20 \mathrm{~cm}$, 17,03 pieds /ha pour $20-40 \mathrm{~cm}$ et 8,26 pieds / ha pour la hauteur supérieure à $40 \mathrm{~cm}$. En zone nord soudanienne, $P$. reticulatum ne présente pas de différence statistique de densité $(\mathrm{P}>0,05)$, entre les différentes classes. L'histogramme de la régénération de $P$. reticulatum en zone sub sahélienne et sud soudanienne affiche une forme en « $\mathrm{L}$ » incurvée et en zone nord soudanienne une allure de «plateau». P. thonningii présente une évolution de la régénération en forme de droite descendante à forte pente.

Chez le genre Piliostigma les classes de régénération sexuée montrent une liaison étroite entre elles par rapport aux effectifs des jeunes plants (Tableau 4). Les coefficients de corrélation de Pearson varient de 0,22 à 0,50 au seuil de $P=0,01$. La classe $[0-20 \mathrm{~cm}$ [correspond à la phase d'établissement de la régénération. C'est au niveau des phases [0-20 $\mathrm{cm}$ [ et supérieur à $40 \mathrm{~cm}$ que nous constatons, hormis en zone nord soudanienne, une baisse d'environ $50 \%$ de la régénération naturelle chez les Piliostigma. Les classes [20$40 \mathrm{~cm}$ [ et Supérieur à $40 \mathrm{~cm}$ correspondent à une phase de survie et de croissance dans la perpétuation des peuplements de Piliostigma.

\section{Piliostigma et pression anthropique}

Piliostigma est une espèce pionnière et aussi un genre persistant que l'on rencontre dans les vieilles jachères. Ces jachères sont aussi régulièrement parcourues par des feux de brousse sauvages. En zone sub sahélienne, la totalité des formations de Piliostigma sont utilisées comme zone de pâturage et parcourues par les feux de brousse. Il en est de même dans la zone sud soudanienne. En zone nord soudanienne, $18,8 \%$ des formations de
Piliostigma sont seulement pâturées contre $81,2 \%$ qui sont pâturées et régulièrement parcourues par les feux de brousse.

L'intensité des actions humaines en termes de pâture par le bétail, de feux de brousse, d'écorçage et de coupe de bois (Photos1 et 2), sur les peuplements de Piliostigma est très variable selon les zones phytogéographiques. En zone sub sahélienne, elle est élevée sur l'ensemble des formations. En zone sud soudanienne, elle est élevée dans $38,3 \%$ des formations et jugée modérée dans $61,7 \%$ des cas. En zone nord soudanienne la pression humaine est forte dans $64,7 \%$ des cas, modérée dans $29,1 \%$ des cas et jugée faible dans $6,2 \%$ des cas. La coupe à ras du sol de pied, la coupe sélective de branche et l'écorçage de branche sont les actions humaines sur les individus du genre Piliostigma. Ces diverses actions ont une ampleur variée suivant les zones. En zone sub sahélienne, l'écorçage est le type de pression anthropique le plus fréquemment rencontré (14,9\%), au niveau des sujets de $P$. reticulatum, contre $9,7 \%$ de coupe. C'est dans cette zone qu'on rencontre le plus de pieds sains de $P$. reticulatum soit $67,7 \%$. En zone sud soudanienne, l'espèce $P$. reticulatum est plus épargnée par l'activité humaine $(22,5 \%)$ que $P$. thonningii (14\%). Les coupes observées sur les sujets de $P$. reticulatum et $P$. thonningii, interviennent, le plus souvent, lors de l'installation de nouveaux champs. C'est en zone nord soudanienne que Piliostigma, est l'objet des plus importantes agressions humaines. Dans cette zone, $P$. reticulatum est plus épargnée $(45,8 \%)$ que $P$. thonningii $(21,1 \%)$. Cette forte pression anthropique a des influences sur les mécanismes de régénération des Piliostigma dans les jachères. Les plantules annuelles disparaissent au dessus du sol dès le mois de décembre avec la saison sèche et les feux de brousse. La morphologie racinaire chez le genre Piliostigma indique un pivot racinaire généralement tubérisé (Photo 3). La tubérisation de la partie souterraine est liée au degré d'anthropisation des Piliostigma. 
Tableau 1: Densité des peuplements (moyenne \pm écartype) des différentes zones phytogéographiques de l'étude.

\begin{tabular}{lcc}
\hline Sites & Espèce & Densité (nombre de pieds/ha) \\
\hline Sub sahélienne & P. reticulatum & $607,14 \pm 27,45$ \\
& & \\
Nord soudanienne & P. reticulatum & $269,77 \pm 12,23$ \\
& P. thonningii & $69,93 \pm 6,98$ \\
& & \\
Sud soudanienne & P. reticulatum & $129,23 \pm 10,67$ \\
& P. thonningii & $499,07 \pm 42,23$ \\
\hline
\end{tabular}

Tableau 2: Evolution de la hauteur et du diamètre moyens chez le genre Piliostigma suivant différentes zones phytogéographiques (moyenne \pm écartype).

\begin{tabular}{llcc}
\hline $\begin{array}{l}\text { Zone } \\
\text { phytogéographique }\end{array}$ & Espèce & Hauteur totale $(\mathbf{c m})$ & Diamètre base $(\mathbf{c m})$ \\
\hline Zone sud soudanienne & P. reticulatum & $181,86 \pm 74,89_{\mathrm{a}}$ & $3,26 \pm 1,71_{\mathrm{a}}$ \\
& P. thonningii & $232,74 \pm 82,59_{\mathrm{b}}$ & $4,68 \pm 2,59 \mathrm{~b}_{\mathrm{b}}$ \\
Zone nord soudanienne & P. reticulatum & $174,82 \pm 69,11_{\mathrm{a}}$ & $3,36 \pm 1,59_{\mathrm{a}}$ \\
& P. thonningii & $206,22 \pm 69,81_{\mathrm{c}}$ & $4,02 \pm 1,85_{\mathrm{b}}$ \\
Zone sub sahélienne & P. reticulatum & $213,96 \pm 76,96_{\mathrm{c}}$ & $5,39 \pm 2,30_{\mathrm{c}}$ \\
\hline \multicolumn{2}{l}{ Par colonne, les lettres en indice différentes indiquent une différence significative de moyenne $(\mathrm{P}<0,05)}$.
\end{tabular}

Tableau 3: Densité de la régénération (nombre de pieds/ha) des espèces du genre Piliostigma selon différentes zones phytogéographiques.

\begin{tabular}{lcc}
\hline Zone phytogéographique & Espèce & Densité \\
\hline Zone sud soudanienne & $P$. reticulatum & $25,68 \pm 2,97_{\mathrm{a}}$ \\
& P. thonningii & $68,39 \pm 8,69_{\mathrm{b}}$ \\
Zone nord soudanienne & P. reticulatum & $39,42 \pm 5,74_{\mathrm{c}}$ \\
& P. thonningii & $03,03 \pm 1,14_{\mathrm{d}}$ \\
Zone sub sahélienne & P. reticulatum & $16,57 \pm 3,25_{\mathrm{e}}$ \\
\hline Par colonne les lettres en indice différentes indiquent une différence significative de moyenne $(\mathrm{P}<0,05)$.
\end{tabular}

Tableau 4: Corrélations entre les classes de hauteur de régénération.

\begin{tabular}{llll}
\hline & $\mathbf{0 - 2 0} \mathbf{~ c m}[$ & {$[\mathbf{2 0 - 4 0} \mathbf{~ c m}[$} & Sup. à $\mathbf{4 0 c m}$ \\
\hline$[0-20 \mathrm{~cm}[$ & 1,00 & & \\
{$[20-40 \mathrm{~cm}[$} & $0,46^{* *}$ & 1,00 & \\
Sup. à $40 \mathrm{~cm}$ & $0,22^{* *}$ & $0,50^{* *}$ & 1,00 \\
\hline${ }^{* *} \mathrm{P} \leq 0,05$ & & &
\end{tabular}



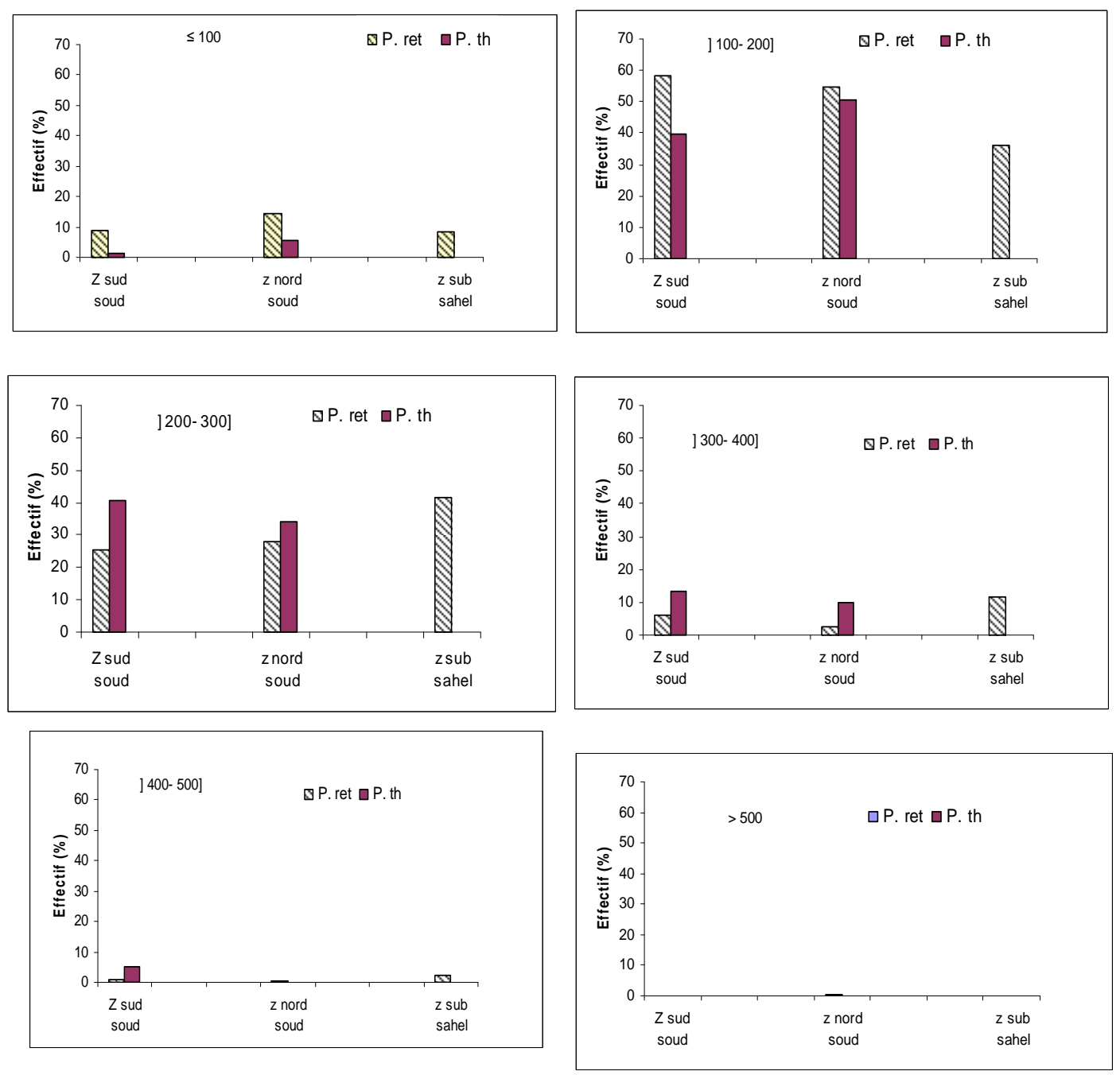

Figure 2: Structure en hauteur des populations de Piliostigma . P. ret = Piliostigma reticulatum $;$ P. th $=$ Piliostigma thonningii.

\section{DISCUSSION}

L'homme perturbe la dynamique des peuplements de Piliostigma par des actions diverses : la coupe pour le bois, l'installation des champs, la cueillette des feuilles et gousses, l'écorçage pour le cordage et les feux de brousse sauvages (Ouédraogo, 2006 ; Yélémou et al., 2007). De par leur proximité à l'homme, liée à leur place dans les rites et pharmacopée traditionnels et leur rusticité (Yélémou et al., 2007), les espèces du genre Piliostigma sont beaucoup anthropisées. Cependant, le mode de gestion de Piliostigma est variable suivant les zones et les populations. En zone sub sahélienne, les populations pratiquent surtout l'élevage traditionnel avec un important cheptel. L'élevage est confronté à la faiblesse du fourrage herbacé consécutive aux pluies annuelles insuffisantes à irrégulières, et à la rareté du fourrage ligneux. Piliostigma spp, espèce rustique à gousses bien appétées par le bétail (Zoundi et al., 2004), est alors convoitée pour l'alimentation du bétail. C'est en zone sub sahélienne que l'espèce $P$. reticulatum, est la plus épargné par les populations. 

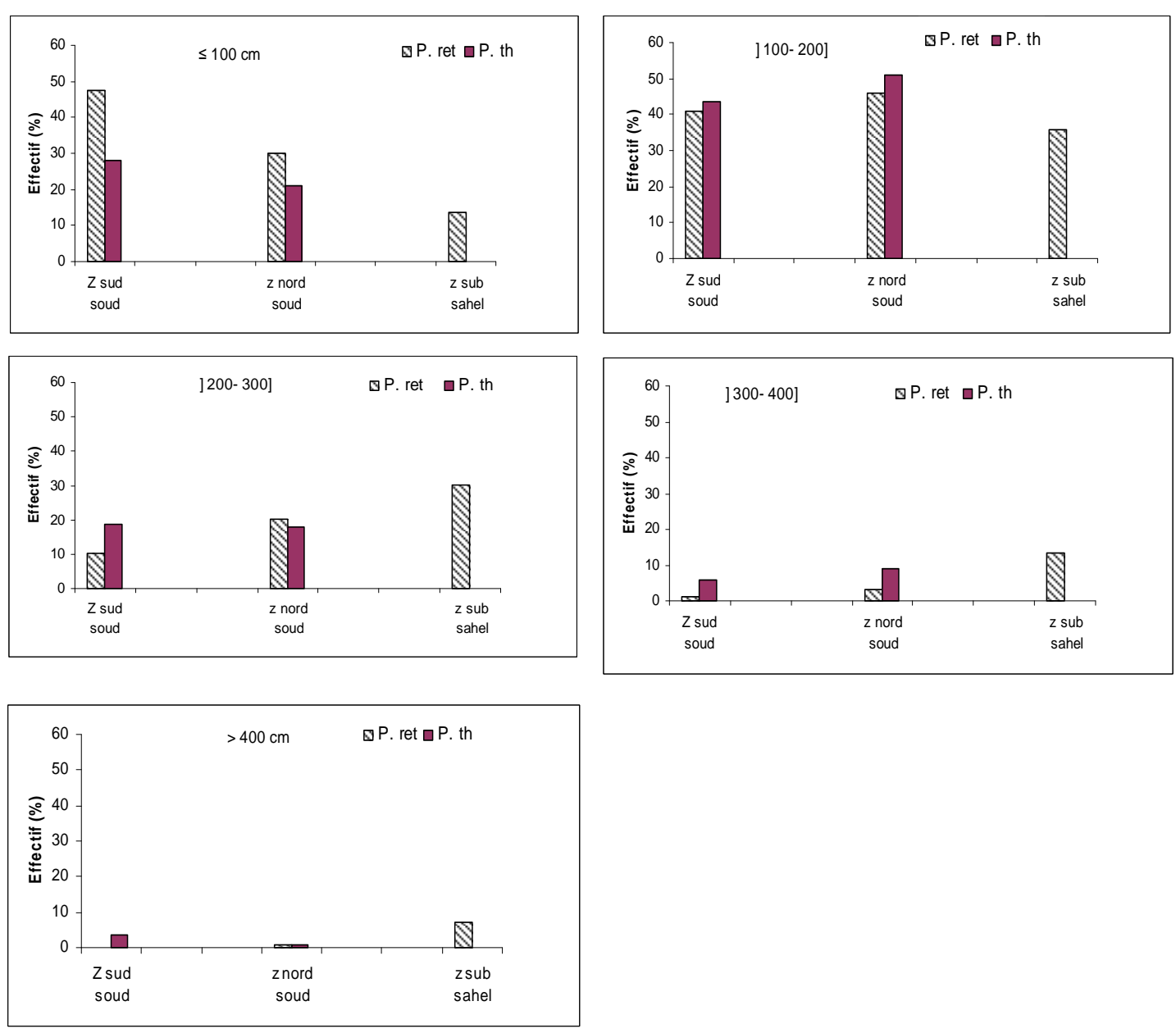

Figure 3: Structure du houppier suivant les zones phytogéographiques. P. ret = Piliostigma reticulatum $; \mathrm{P}$. th = Piliostigma thonningii.

La préservation des pieds de Piliostigma en vue de la production de gousses comme aliment de bétail et son rôle fertilisant et antiérosif, sont les principaux intérêts recherchés dans la gestion de cette espèce. En zone nord soudanienne, zone la plus peuplée du pays, la pression démographique est très forte. Les populations surtout agricoles sont confrontées au problème d'insuffisance des terres et de bois d'énergie. En outre, dans cette zone, les nombreux usages en pharmacopée de Piliostigma, les exposent à des coupes fréquentes. Dans un terroir, lorsque les deux espèces de
Piliostigma sont présentes, la préférence des populations pour la cueillette des feuilles et la coupe du bois de l'écorce, vont pour $P$. thonningii, à cause de ses caractéristiques morphologiques (écorce moins rugueuse, feuilles plus larges et lisses...). La zone sud soudanienne du Burkina Faso, peu peuplée avec de nombreuses terres fertiles (Paré, 2008), est une destination des immigrants ruraux à la recherche de vastes terres cultivables, et c'est également la zone de coupe de bois d'énergie pour l'approvisionnement des centres urbains. 

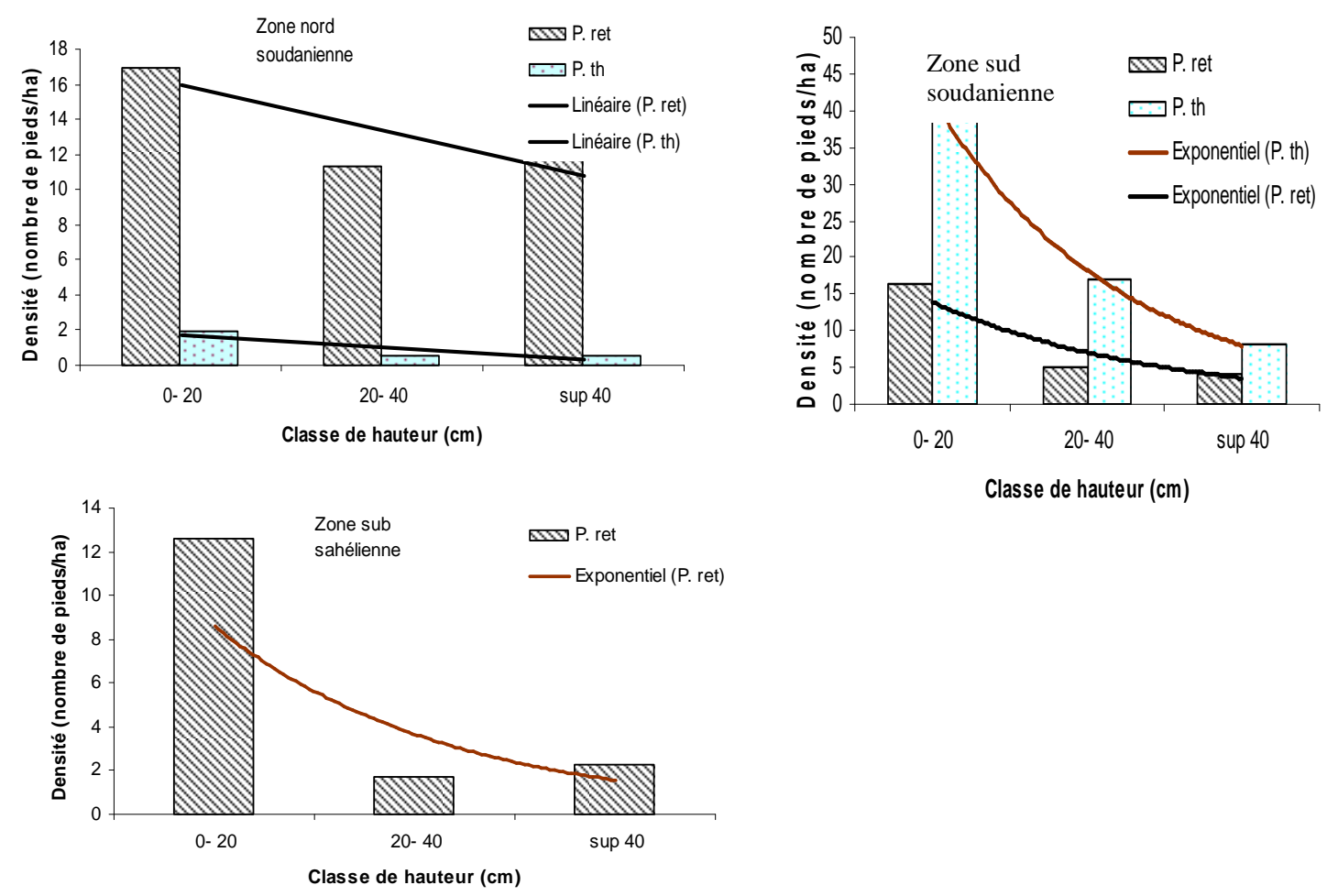

Figure 4: Evolution de la régénération naturelle de Piliostigma spp dans différentes zones phytogéographiques $(\mathrm{P}$. ret $=$ Piliostigma reticulatum $; \mathrm{P}$. th $=$ Piliostigma thonningii) .

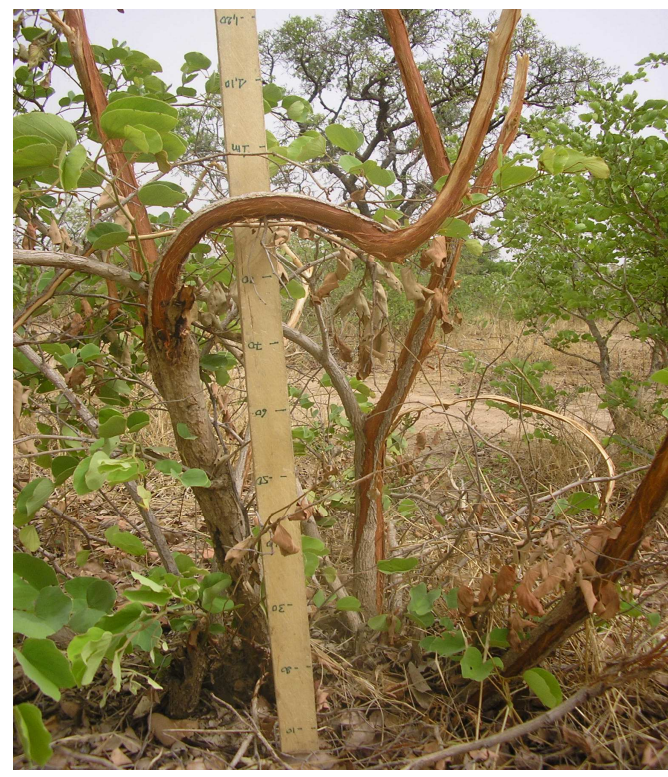

Photo 1: Un pied de $P$. thonningii fortement écorçé avec traces de coupe à la base Photo B. Yélémou 2007.

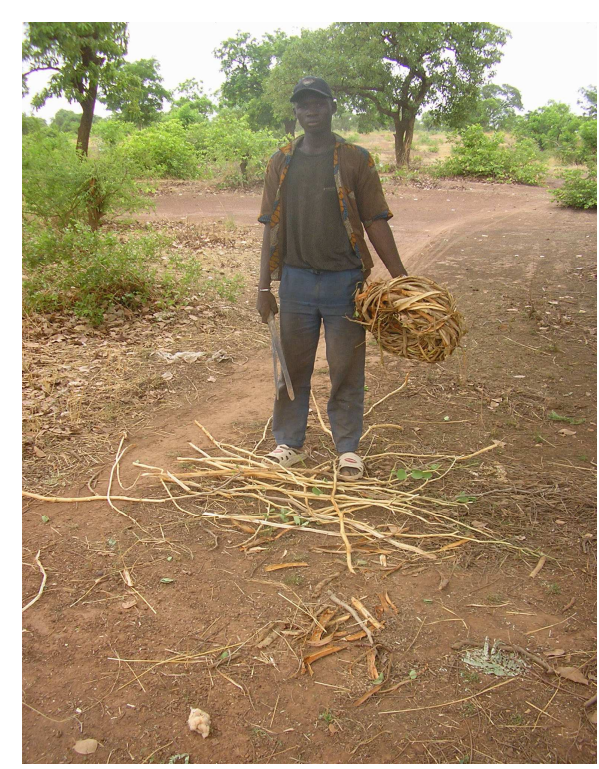

Photo 2: Rouleau d'écorce de P. reticulatum fraîchement coupé Photo B.Yélémou 2007. 


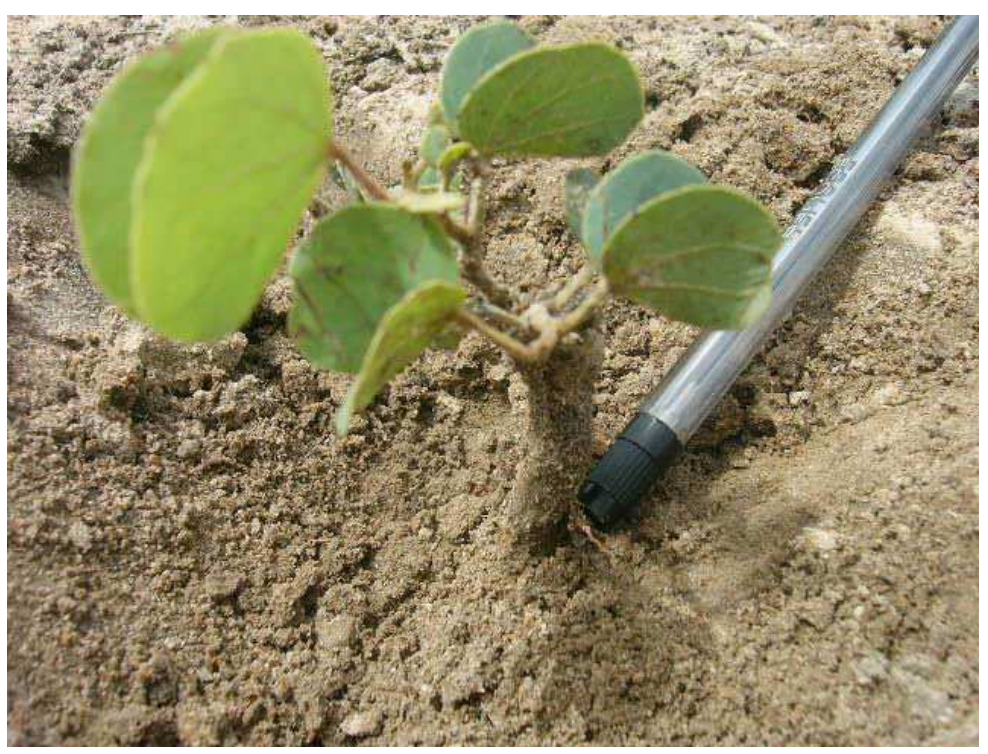

Photo 3: Important développement de la racine par rapport à la partie aérienne de $P$. reticulatum . (Yélémou, 2008).

Les espèces du genre Piliostigma y sont donc abondamment coupées. La production des gousses est fortement dépendante de l'importance du houppier (Yélémou et al., 2009). Dans la zone sub sahélienne, les individus de houppier moyen sont plus abondants ce qui traduit une stabilité dans la dynamique de la production, les vieux pieds à faible production de gousses étant peu représentés. En zone nord soudanienne, sous l'effet de la pression anthropique, les peuplements de Piliostigma connaissent un déclin à partir de la classe de houppier [200-, 300[. Cette faible représentativité des individus de grand diamètre chez les Piliostigma, est liée aux diverses exploitations que connaissent les gros sujets (pour leur fibre, le bois et des besoins en pharmacopée). $P$. thonningii connaît également un déclin de sa population à partir de la classe de houppier [100-200[. A l'action directe de l'homme, s'ajoutent les feux de brousse annuels qui ont des effets néfastes importants sur le développement des plantes (Gignoux et al., 1997 ; Traoré et al., 2008). La pression accrue du milieu sur ces espèces entraîne une perturbation des peuplements de Piliostigma, avec surtout un déclin des semenciers. Dans ces conditions, la reproduction sexuée des espèces du genre Piliostigma, semble inadaptée au contexte actuel. La structure de la hauteur des populations chez les Piliostigma, laisse percevoir une faible proportion des individus pour la classe $<100$ $\mathrm{cm}$. Cela traduit les difficultés d'établissement de la croissance chez ces espèces. Ces difficultés peuvent être liées aux feux de brousse fréquents (Gignoux et al., 1997 ; Setterfield, 2002 ; Ouédraogo et al., 2006), à la sécheresse, aux piétinements et au broutage des animaux (Ky-Dembélé et al., 2007 ; Traoré et al., 2008). Par contre, la classe de hauteur [100-200[comporte le plus important nombre d'individus quelle que soit l'espèce. Cette classe de hauteur [100-200[ serait la hauteur à partir de laquelle, les populations de Piliostigma résistent mieux aux diverses pressions externes ou sont moins exploitées. Cependant, au-delà de $200 \mathrm{~m}$ de hauteur, hormis dans la zone sub sahélienne, la pression sur les peuplements de Piliostigma est importante. A partir de la classe de hauteur [400-500[, quelle que soit l'espèce, les peuplements de Piliostigma connaissent un 
fort déclin. Cette faible hauteur des peuplements de Piliostigma, pourrait être un mode d'adaptation de ces espèces à leur milieu écologique. La régénération naturelle non assistée des Piliostigma, est confrontée à de nombreuses contraintes d'origine exogène et endogène. Toutes les espèces fourragères sont exposées à une exploitation continue par les animaux en zone sahélienne (Thiombiano et al., 2003). La régénération naturelle non assistée de plusieurs espèces connaît des difficultés qui sont surtout liées aux conditions stationnelles (Thiombiano et al., 2003). En effet les feux de brousse annuels fréquents détruisent les semences, les plantules annuels (Gignoux et al., 1997 ; Hoffmann, 1998 ; Setterfield, 2002 ; Ouédraogo et al., 2006 ; Vieira et al., 2007 ; Muoghalu, 2007). La faible régénération des espèces du genre Piliostigma pourrait être aussi due aux nombreuses attaques des gousses par des insectes. En effet de nombreux insectes sont observés sur les pieds de Piliostigma, surtout au moment de la floraison-fructification (Yélémou et al., 2008). De nombreuses gousses de ces espèces sont perforées et même réduites en poudre en fin de maturation. Les parasites des graines détruisent la partie vitale de la semence.

$P$. thonningii présente la plus forte régénération naturelle en zone sud soudanienne et $P$. reticulatum, en zone nord soudanienne. Ces zones correspondent respectivement aux aires de distribution naturelle des deux espèces. L'aire de distribution offre pour l'espèce les conditions idéales de température, d'humidité et de sol pour son plein épanouissement. La structuration de la régénération en classe de hauteur met en évidence l'existence des contraintes réelles d'installation de la régénération naturelle chez les espèces du genre Piliostigma dans les zones d'étude. Ces difficultés s'expliquent surtout par la pression pastorale et les feux de brousse annuels. Le mode d'utilisation des terres affecte toutes les classes de régénération des plantes et plus particulièrement des petites classes (Traoré et al., 2008). Il faut aussi noter que le bétail demeure un des principaux facteurs favorisant la régénération du genre Piliostigma. En effet, il assure la dissémination des graines et améliore le taux de germination in situ grâce au transit intestinal. L'installation de la régénération nécessite aussi une humidité régulière. Plusieurs auteurs indiquent que l'irrégularité des précipitations en zone sahélienne constitue une contrainte majeure à la régénération des espèces (Gampiné et Boussim, 1995 ; Thiombiano et al., 2003). En zone nord soudanienne, la densité des individus ne diffère pas sensiblement suivant les classes de hauteurs, quelles que soient les espèces. Dans cette zone, 35\% de nos sites d'étude ont pu être protégés des feux de brousse au cours des trois dernières années. Le régime de zones protégées permet une structure stable des plantes avec une abondance de la régénération (Traoré et al., 2008). Des résultats identiques ont été obtenus dans l'étude des Combretaceae et Caesalpiniaceae en zones protégées et zones anthropisées (Gampiné et Boussim, 1995). La protection partielle contre les feux de brousse expliquerait l'équilibre de la régénération chez les deux espèces de Piliostigma. Le genre Piliostigma a une capacité d'adaptation aux feux à cause de la morphologie fonctionnelle de ses plantules. Cependant, la réelle menace pour la régénération serait les feux de brousse qui bien souvent surviennent en saison sèche au moment où la plante est déjà dans un état de stress hydrique très avancé.

\section{Conclusion}

Malgré les conditions climatiques arides, c'est en zone sub sahélienne que les formations à Piliostigma sont mieux conservées, en relation avec les besoins des populations rurales. La gestion traditionnelle des Piliostigma n'est pas en adéquation avec les difficultés d'installation de ces espèces, ce qui à long terme pourrait poser le problème de déclin de ces espèces. Les Piliostigma connaissent une régénération relativement faible du fait de la combinaison des facteurs anthropiques et de la rigueur du climat. Cette faiblesse de la régénération sexuée est paliée 
par la dynamique de sa régénération végétative. Les espèces du genre Piliostigma présentent des dispositifs anatomiques leur permettant de bien tirer partie de la forte pression anthropique subie: dynamisme et enfouissement du collet en profondeur; réveil physiologique des graines par le feu, écorce et feuilles épaisses. Cependant, pour une meilleure expression génétique de ces espèces, leur reproduction sexuée mérite d'être soutenue par des actions de reboisement ou de protection des individus fragiles c'est-àdire de 0 à $20 \mathrm{~cm}$ de hauteur.

\section{REFERENCES}

Bationo BA, Ouédraogo SJ, Guinko S. 2001. Stratégies de régénération naturelle de Detarium microcarpum Guill. et Perr. dans la forêt classée de Nazinon (Burkina Faso). Fruits, 4: 271-85.

Bationo BA, Ouédraogo SJ, Somé AN, Pallo F, Boussim IJ. 2005. Régénération naturelle d'Isoberlinia doka Craib. et Stapf. dans la forêt classée du Nazinon (Burkina Faso). Cah. Agr., 3: 297-303.

Diedhou S, Dossa EL, Badiane AN, Diedhou I, Séné M, Dick RP. 2009. Decomposition and spatial microbial heterogeneity associated with native shrubs in soils of agroecosystems in semiarid Senegal. Pedobiologia, 52: 273-286.

Dossa EL, Khouma M, Diedhou I, Séné M, Kizito F, Badiane AN, Samba SAN, Dick RP. 2009. Carbon, nitrogen and phosphorus mineralization potential of semiarid Sahelian soils amended with native shrub residues. Geoderma, 148: 251-260.

Fontes J, Guinko S. 1995. Carte de la végétation et de l'occupation du sol du Burkina Faso. Ministère de la Coopération Française, projet campus (88 313 101), 67 p.

Gampine D, Boussim IJ. 1995. Etude de la contrainte à la régénération de neuf espèces ligneuses au Burkina Faso. Etudes Flor. Vég. Burkina Faso, 1: 3-16.
Gignoux J, Clobert J, Menaut JC. 1997. Alternative fire resistance strategies in savanna trees. Oecologia, 110: 576-583.

Hoffmann AW. 1998. Post-burn reproduction of woody plants in a neotropical savanna, the relative importance of sexual and vegetative reproduction. J. Applied Ecol., 35: 422-433.

Kaboré A, Tamboura HH, Belem AMG, Traoré A. 2007. Traitements ethnovétérinaires des parasitoses digestives des petits ruminants dans le plateau central du Burkina Faso. Int. J. Biol. Chim. Sci., 1: 297-304.

Ky-Dembelé C, Tigabu M, Bayala J, Ouédraogo SJ, Odén PC. 2007. The relative importance of different regeneration mechanisms in a selectively cut savanna-woodland in Burkina Faso, West Africa. For. Ecol. Manag., 243: 2838.

Lufala A, Diedhou I, Ndiaye NAS, Séné M, Kizito F, Dick RP, Noller JS. 2009. Allometric relationships and peak-season community biomass stocks of Native shrubs in Senegal's Peanut Basin. Journal of Arid Environments, 73: 260-266.

Muoghalu JI. 2007. Tree species population dynamics in a secondary fores tat Ile-Ife, Nigeria after a ground fire. Afri. Jour Ecol., 45: 62-71.

Ouédraogo A. 2006. Diversité et dynamique de la végétation ligneuse de la partie orientale du Burkina Faso. Thèse de Doctorat unique, Université de Ouagadougou, $195 \mathrm{p}$.

Ouédraogo A, Thiombiano A, Hahn-Hadjali K, Guinko S. 2006. Régénération sexuée de Boswellia dalzielii Hutch., un arbre médicinal de grande valeur au Burkina Faso. Bois For. Trop., 289: 41-48.

Paré S. 2008. Land use dynamics, tree diversity and local perception of dry forest decline in southern Burkina Faso, West Africa. Doctoral thesis 78, Swedish University of Agricultural Sciences, Umea.

Setterfield SA. 2002. Seedling establishment in an Australian tropical savannah: effects 
of seed supply, soil disturbance and fire. $J$ Apply Ecol., 39: 949-956.

Thiombiano A. 1996. Contribution à l'étude des Combretaceae dans les formations végétales de la région Est du Burkina Faso. Thèse de Doctorat 3è cycle, Université de Ouagadougou, $220 \mathrm{p}$.

Thiombiano A, Wittig R, Guinko S. 2003. Conditions de la multiplication sexuée chez des Combretaceae du Burkina Faso. Rev Ecol., 58: 361-379.

Traoré S, Kaboré O, Millogo-Rasolodimby J, Thiombiano L, Guinko S. 2008. Impact of protected areas and land use on regeneration of Acacia woodland's in Eastern Burkina Faso. Flora et Vegetatio Sudano-Sambesica, 11: 17-24.

Vieira DLM, Scariot A. 2007. Principles of natural regeneration of tropical dry forests for restoration. Restor. Ecol., 14: 11-20.

Yélémou B, Bationo B, Yaméogo G, Millogo-Rasolodimby J. 2007. Gestion traditionnelle et usage de Piliostigma reticulatum (D.C.) Hochst., dans le plateau central du Burkina Faso. Bois For. Trop., 291: 55-65.
Yélémou B, Yaméogo G, Bationo BA, Millogo/Rasolodimby J, Hien V. 2008. Biologie florale et mode de reproduction sexuée de Piliostigma reticulatum, (DC) Hochst. Int. J. Biol. Chem. Sci., 2: 281291.

Yélémou B, Zougmoré R, Bationo BA, Millogo/Rasolodimby J, Hien V. 2009. Phenology and fruit production of Piliostigma reticulatum (DC), Hochst., an agroforestry forage species in the Sahel. Cam. Jour. Exp. Biol., 5: 10-20.

Yélémou B. 2010. Biologie et écologie des espèces du genre Piliostigma et leur contribution à la dynamique de la matière organique des sols en zone sahélosoudanienne au Burkina Faso. Mémoire Thèse unique de Doctorat, Université de Ouagadougou, $150 \mathrm{p}$.

Zoundi SJ, Tiendrébéogo JP, Traoré S, Lalba A. 2004. Embouche ovine en zone soudano sahélienne du Burkina Faso. Une alternative pour une amélioration de la sécurité alimentaire. RASPA, 2: 170-174. 